\title{
An alternative channel for type la supernovae explosions
}

\section{Emanuela Chiosi, Cesare Chiosi,}

Dipartimento di Fisica e Astronomia, Università di Padova, vicolo Osservatorio 3, 35122

Padova, Italy

\section{Marina Orio,*}

INAF-Padova, vicolo Osservatorio 5, 35122 Padova, Italy, and Department of Astronomy, 475

N. Charter Str, University of Wisconsin, Madison WI 53706, USA

E-mail: marina.orio@oapd.inaf.it

\section{Lorenzo Piovan and Patrizia Trevisan}

Dipartimento di Fisica e Astronomia, Università di Padova, vicolo Osservatorio 3, 35122

Padova, Italy

\begin{abstract}
The initial reaction triggering a thermonuclear supernova explosion is thought to be a ${ }^{12} \mathrm{C}+{ }^{12} \mathrm{C}$ reaction in the pycnonuclear regime. Such a high density is required, that it can be reached only in a white dwarf that is very compact and massive, close to the Chandrasekhar mass. I report here on work we published a short time before this conference (Chiosi et al. 2015), in which we explored the possibility that other nuclear reactions may occur in the pycnonuclear regime, namely those involving traces of hydrogen and helium, that do not produce sufficient energy to allow the white dwarf to explode, but manage to heat it and change its structure, eventually leading to a type Ia supernova explosion in a sub-Chandrasekhar mass white dwarf. This channel may even lead to the supernova explosion of isolated stars.
\end{abstract}

The Golden Age of Cataclysmic Variables and Related Objects - III, Golden2015

7-12 September 2015

Palermo, Italy

\footnotetext{
* Speaker.
} 


\section{A "crazily high" density?}

Why are we talking about near-Chandrasekhar mass when discussing the scenarios that lead to type Ia SNe? Near-Chandrasekhar mass implies that the white dwarf density (hereafter WD) is sufficiently high to allow ignition of the pycnonuclear reactions thought to trigger type Ia supernovae (hereafter $\mathrm{SNe}$ ) explosions. The initial reactions, involving ${ }^{12} \mathrm{C}+{ }^{12} \mathrm{C}$, occur only at "crazily" high density, about $10^{9} \mathrm{~g} \mathrm{~cm}^{-3}$. Before the explosion, most models foresee a simmering phase that may last even a thousand years; eventually the thermonuclear regime is reached because of the extreme heat that is released, and the explosion may occur. In Chiosi et al. (2015) we explored the possibility that previously unforeseen nuclear reactions lift the pycnonuclear regime before ${ }^{12} \mathrm{C}$ is ignited, allowing explosion in sub-Chandrasekhar WDs.

\section{Progenitors' scenarios}

The scenarios that have been proposed to explain SNe Ia explosions can be broadly divided into the following classes:

1. Single degenerate: these models involve a massive, most likely fast-rotating WD. The $\mathrm{SNe}$ Ia rates may be difficult to reproduce with only single degenerate systems (see e.g. the case of recurrent novae, Shafter et al. 2015) and another hurdle is explaining the very small hydrogen content (we only have upper limits from the observations).

2. Double degenerate: with these models it is the explosion itself that is more difficult to explain. Moreover, what happens during the merger has not well explored (however, see Zhu et al. 2015 for recent new results). Many authors noticed that the merger must happen under special circumstances or it would overproduce the SNe Ia rates. Probably, mergers more often produce neutron stars by accretion induced collapse.

3. Core degenerate: such models involve an old WD and a post-AGB core, merging when the secondary is still in the planetary nebula phase (see Tsebrenko \& Soker 2015).

4. A double detonation due to helium accretion from another WD. When helium detonates, it triggers explosive $\mathrm{C}$ burning in the companion (Shen \& Bildsten 2014). Helium detonation may also trigger the explosive $\mathrm{C}$ burning on the same WD (Shen \& Bildsten 2009).

5. WD+WD collisions in triple systems: this is a model by Kushnir et al. (2013). Collisions in triple systems must often occur, so when two WDs collide, they may merge and explode as type Ia SNe (Kushnir \& Katz 2014).

We must bear in mind that in single star evolution CO WDs are only born with $\mathrm{m} \leq 1.05 \mathrm{M}_{\odot}$ (see recent results by Doherty et al., 2015). The models foresee a small population of $\mathrm{NeO}$ WDs, or $\mathrm{CO}$ WDs with a $\mathrm{NeO}$ layer on top. Doherty et al. (2015) indicate a range 6.6-7.5 $\mathrm{M}_{\odot}$ for the progenitors of "hybrid" WDs and a 8.2-9.5 $\mathrm{M}_{\odot}$ range for the progenitors of $\mathrm{NeO}$ WDs. Kepler et al. (2015) find that binary evolution produces a significant number of low mass WD's, but there are also more numerous WDs well above $1.1 \mathrm{M}_{\odot}$ than stellar evolution theory predicts. Are these all $\mathrm{NeO}$ WDs? Since ONe WDs are rare, it is likely that this high mass range includes also many WDs of CO, whose initial mass has been significantly increased by accretion in close binaries. Unfortunately, spectra can only be obtained for a few objects of Kepler et al.' s sample, so for the time being we cannot consider this as evidence in favor of the single degenerate model. 
As it has been often reminded during this conference, during accretion and hydrogen burning in a WD, we expect a phase of supersoft X-ray (or extreme UV). In X-rays, such objects are detectable at large distances if there is little absorption (both along the line of sight and intrinsic in the binary, like may be the case of symbiotics). Statistics of supersoft X-ray sources have also been invoked way to understand the importance of single degenerate systems to the SNe Ia rate.

\section{Supernovae and nickel production}

Over a hundred species are involved in the SN Ia nuclear reactions, and more than a thousand different reactions are possible. However, without ${ }^{12} \mathrm{C}+{ }^{12} \mathrm{C}$ yielding ${ }^{20} \mathrm{Ne}$ and an alpha particle, or ${ }^{23} \mathrm{Na}$ and a proton, or ${ }^{23} \mathrm{Mg}$ and a neutron, the series of reactions leading to the explosion are not possible.

The INTEGRAL results on the cobalt $\left({ }^{56} \mathrm{Co}\right)$ lines detected at 847 and $1238 \mathrm{keV}$ in a type Ia SN (Churazov et al. 2014) have proved that the thermonuclear explosion is the correct explanation for the SN Ia phenomenon. The light of a SN Ia is thought to be powered by the radioactive decay of nickel produced in the explosion. In fact, nickel is short lived and the lines due to its decay are difficult to observe due to absorption by the ejecta, however when nickel decays into cobalt (later cobalt will decay into iron), cobalt is much longer lived (for one type of decay the half life is 11.3 days, for the other 77.12 days).

\section{What we know of the progenitors}

Although the progenitor of a SN Ia has never been observed, there are observational facts in favor of a mix of single and double degenerate progenitors. Hubble Space Telescope deep preexplosion imaging of the site of SN 2011fe rules out evolved companion stars with $\mathrm{M}>3.5 \mathrm{M}_{\odot}$ (Li et al. 2011). On the other hand, Marion et al. (2016) detected excess blue light in the explosion of SN $2012 \mathrm{cg}$, consistent with the predictions of the impact of the SN on a non-degenerate companion.

Evidence of a dependence of SNe Ia star formation rate has been known for years, and studies of the "delay time" between star formation and SNe Ia explosions in galaxies of different morphological classes indicate that the progenitors cannot be only old stars (see, e.g., review by Greggio 2008 and references therein). Milne (2015) and Foley (2016) divide SNe Ia according on UV colors; there is also evidence of a separate class of underluminous class of "SNe Iax" that show interactions with the circumstellar medium (see Patat et al. 2011, 2012). The Iax are thought to belong to an old population and to be single degenerate systems. The jury is still out for the other SNe Ia.

Scalzo et al. (2014) have derived statistics on the distribution of events by WD mass, assuming it is proportional to the inferred mass of nickel ejecta: they assumed (as it is commonly done) that the nickel mass powering the light curve is related to the light curve width and the absolute magnitude of the supernova at maximum. These authors find both super- and sub-Chandrasekhar mass $\mathrm{SNe}$ Ia, and the latter should be quite common. 


\section{Five regimes of nuclear reactions}

1. The first regime is that of the "classic" thermonuclear reactions in an ideal gas.

2. In a medium that is between a liquid and a lattice, thermonuclear reactions occur with strong electron screening.

3. The thermo-pycno-nuclear regime follows after a further step towards crystallization. The reactions occur between highly thermally excited bound nuclei, and are enhanced by the charge screening electron background.

4. Before reaching the melting temperature, there is a thermally enhanced pycnonuclear regime.

5. Finally, we have the purely pycno-nuclear, "T=0", WD crystallized or solid medium in which reactions between closest pairs occur by tunneling effect and there is no temperature dependence. The density determines whether the reactions occur or not.

A near-Chandrasekhar mass white dwarf has a radius close to $2000 \mathrm{~km}$. The gravitational binding energy is about $2.6 \times 10^{51} \mathrm{erg}$, and such energy must be released if the whole star has to burn. Assuming a pure ${ }^{12} \mathrm{C}$ composition and burning ending with only ${ }^{56} \mathrm{Ni}$, the q value is $\mathrm{q} \simeq 9.2 \times 10^{17} \mathrm{erg} \mathrm{g}^{-1}$. A much more detailed calculation gives lower values for both the $\mathrm{q}$ value (only a few $10^{15} \mathrm{erg} \mathrm{g}^{-1}$ ) and of the gravitational energy, but confirms that carbon burning can unbind a massive $\mathrm{CO}$ white dwarf. Because the Chandrasekhar mass implies infinite density, and when matter is accreted the density of the whole white dwarf immediately "adjusts itself" to the new total mass, close to Chandrasekhar mass a WD reaches the density for the pycnonuclear reaction involving ${ }^{12} \mathrm{C}$.

\section{Are other reactions possible?}

How much hydrogen (or helium) can be left over in a CO WD without complete levitation to the surface? So far, model calculations were stopped before finding out, because the existence of impurities of hydrogen and helium was not thought to be interesting. Furthermore, we know from observations of WD atmospheres that a large amount of the residual hydrogen and helium levitates to the surface and sedimentation in a superficial layer follows. However, at the end of AGB phase, the hydrogen abundance in mass is $\mathrm{X}(\mathrm{H}) \simeq 10^{-20}$ while the helium mass abundance is $\mathrm{X}(\mathrm{He}) \simeq 10^{-6}$ (see extensive discussion in the original Chiosi et al.'s 2015 paper). It is conceivable that traces of these impurities remain in the core.

In a recently published paper by Chiosi et al. (2015) we showed that the impurities may play a very important role and be a driving force towards thermonuclear explosions of WDS. Even with extremely low abundance of hydrogen (or helium), the reaction ${ }^{1} \mathrm{H}+{ }^{12} \mathrm{C}=>{ }^{13} \mathrm{~N}+\gamma+1.95 \mathrm{MeV}$ and a similar reaction with helium, can be ignited. In a massive $\left(\mathrm{m} \geq 1.2 \mathrm{M}_{\odot}\right)$ CO WD this may even occur at the beginning of the cooling phase. We do not know whether we reach this high mass in a binary this early stage, however, even more important is the likely possibility that hydrogen is ignited in the thermo-pycnonuclear regime for WD mass $\geq 1 \mathrm{M}_{\odot}$, or in the pycnonuclear regime for WD mass $\geq 0.8 \mathrm{M}_{\odot}$ exceeding the WD luminosity, after a time that is proportional to the WD mass (see Fig.1). Similar results are found for helium. 
We note in passing that the possible discovery of the coldest WD ever, of $1 \mathrm{M}_{\odot}$ and most likely already undergoing Debye cooling (Kaplan et al. 2014), should pose limits to the hydrogen impurities' abundance (Kaplan's WD has not exploded...), so perhaps we can rule out the lowest WD mass range and the $0.8 \mathrm{M}_{\odot}$ limit is only theoretical.

\section{Calculations of new pycnonuclear reaction rates}

New calculations of pycnonuclear reactions rates of ${ }^{1} \mathrm{H}+{ }^{12} \mathrm{C}$ and ${ }^{4} \mathrm{He}+{ }^{12} \mathrm{C}$ have been presented by Chiosi et al. (2015). The first step for these calculations was a formalism known as "modified harmonic oscillator". Then, following more complex formalism by Salpeter \& van Horn (1969), Kitamura et al. (2000) and Gasques et al. (2005) the reactions involving impurities have been calculated. The important parameters are: a) the inter-ionic distance, b) G, the Coulombian coupling parameter ratio of Coulomb to internal energy, and c) the liquefaction, Debye, and melting temperatures of the nuclei, including the effect of the local density. Chiosi et al. (2015)'s formalism reproduces the results of the other authors and has extended the calculations to reactions with the helium and hydrogen impurities , including also on the local density of the elements that "pollute" the WD. The work also includes a new model (modified harmonic oscillator, or MHO, formalism), specifically studied to account for reactions involving lighter ions of hydrogen or helium in the WD CO ion lattice. The new pycnonuclear energy generation rates have been calculated following more complex prescriptions for a one component plasma (OCP) and for a multi-component plasma (MCP). In Fig.1, we show the time necessary to reach the WD luminosity for a given WD mass, only by pycnonuclear reactions due to hydrogen impurities, that are ignited at much lower density than the ${ }^{12} \mathrm{C}+{ }^{12} \mathrm{C}$ reactions. The time to exceed the WD luminosity may be shorter than the age of the Universe even for a WD of mass as low as $0.8 \mathrm{M}_{\odot}$, although this may occur at a time the WD is close to crystallization. The red line in Fig. 1 represents the luminosity evolution (in practice, it is a cooling curve) of a "typical" WD of the given mass. the blue curves of Fig. 1 represent the energy generation rate for a given abundance by mass of hydrogen in the white dwarf interior. The time for the intersection of the two curves decreases with increasing white dwarf mass, and with the abundance of impurities.

The pycnonuclear reactions involving hydrogen or helium will heat a volume with the dimensions of the free mean path of the conductive electrons, which is much larger than inter-ionic distances. Therefore, the structure of the white dwarf will be altered. When the pycnonuclear energy generation rate is of the same order of magnitude as the white dwarf luminosity, the white dwarf may be heated to a point that the crystallization of the white dwarf is partially reversed. Then, the ${ }^{12} \mathrm{C}+{ }^{12} \mathrm{C}$ reactions can be ignited in a thermonuclear regime, needing a much lower density and related lower white dwarf mass.

\section{Re-examining the scenarios}

In Chiosi et al. (2015) we have shown that there is a concrete possibility that the WD is heated and the pycnonuclear regime is lifted, so that we do not need to ignite ${ }^{12} \mathrm{C}$ reaction in pycnonuclear regime to obtain a supernova. In the thermonuclear regime, the ${ }^{12} \mathrm{C}+{ }^{12} \mathrm{C}$ reactions and then the rest 

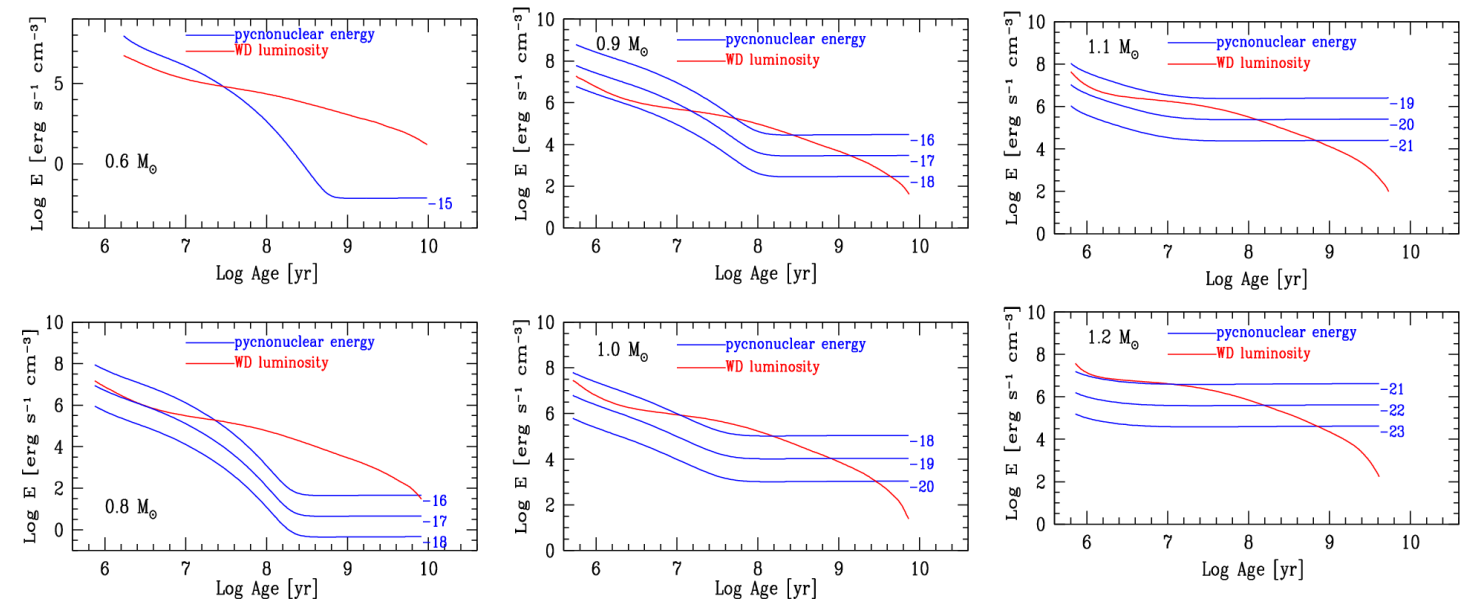

Figure 1: Relative evolution of the white dwarf luminosity and the pycnonuclear energy generation rate due to the burning of ${ }^{1} \mathrm{H}+{ }^{12} \mathrm{C}$, for different white dwarf masses. The pycnonuclear energy generation rate has been calculated for three different abundances (by mass) of hydrogen in each case. Note that the upper blue line crosses the red line twice for some of the highest abundances. Note also that the higher is the mass of the white dwarf, the lower the hydrogen abundance necessary to cross the red line before the age of the Universe.

of the reactions will follow, eventually leading all the way to iron nucleosynthesis, at much lower density (so, with much smaller WD mass!) than in the pycnonuclear regime.

From the analysis of $\mathrm{Na}$ absorption lines, evidence of circumstellar material and morphology, Tsebrenko \& Soker (2015) concluded that about $20 \%$ of SNe Ia occur in planetary nebulae. They suggested that in binary systems WDs merge with the AGB core of the companion. However, if the impurities' burning is ignited, SNe Ia in planetary nebulae may be explained even as single degenerate WDs exploding at a very young age, practically "in the making", if they are relatively massive, and are immediately heated by the pycnonuclear reactions of hydrogen and helium.

In any case, it is interesting that our new mechanism may explain an evolutionary channel in which the SNe Ia rate naturally depends on the star formation rate, because the more massive the WD is, either by birth or due to accretion in a binary, the sooner the pycnonuclear reactions involving impurities occur.

\section{A path forward}

We found that the impurities may start burning even in the intermediate thermo-pycno-nuclear regime. In any case, the burning involving the impurities would occur at much lower density than the reactions with two ${ }^{12} \mathrm{C}$ nuclei, even when it starts only in the fully pycnonuclear regime. As a result, for masses as low as $0.8 \mathrm{M}_{\odot}$, even a minimal abundance of impurities may cause significant re-heating of the WD before the age of the Universe. The impurities' burning may explain the occurrence of sub-Chandrasekhar explosions as a consequence of stellar evolution, and the dependence of the SNe Ia rate on the star formation rate. It may even be the mechanism eventually leading to SNe Ia exploding in young populations and to the "SNIPs" (supernovae in 
planetary nebulae). The Scalzo et al. (2014) distribution of progenitors' masses, skewed towards the low mass end, is also naturally explained.

The importance of this new physical mechanism and its consequences, of course, must be proved with detailed calculations of WD evolution during sedimentation and burning, and with full-fledged calculations of the explosions that may follow. However, this model appears simpler than others... and should we not apply Occam's razor? In fact, our results on the new pycnonuclear reactions' rates may imply that explosions occur on isolated WDs. Of course, we do know that at least half of all stars are in binaries, but our model also alleviates the main problem of "single degenerates", namely the difficulty of accreting enough mass to exceed $1.35 \mathrm{M}_{\odot}$, implying that sub-Chandrasekhar progenitors are common. Detailed population synthesis calculations may also indicate how significant and relevant the impurities' burning is, for both isolated WDs and/or for single degenerate accretors.

\section{References}

[1] Chiosi, E., Chiosi, C., Trevisan, P., Piovan, L. \& Orio, M. 2015, MNRAS, 448, 2100

[2] Churazov., E., et al. 2014, Nature, 512, 406

[3] Doherty, C.L., et al. 2015, MNRAS, 446, 2599

[4] Foley, R.J., et al. 2016, MNRAS, 461, 1308

[5] Gasques, L.R., et al. 2005, Phys. Rev. C, 72, 025806

[6] Greggio, L. 2008, MNRAS, 406, 22

[7] Kaplan, D., et al. 2014, ApJ, 789, 119

[8] Kepler, S.O., et al. 2015, MNRAS, 446, 4078

[9] Kitamura H., 2000, ApJ, 539, 888

[10] Kushnir, D., Katz, B., Dong, S., Livne, E. \& Fernandez, R. 2013, ApJ, 778, L37

[11] Kushnir, D., \& Katz, B. 2014, ApJ, 785, 124

[12] Li, W., et al. 2011, Nature, 480, 348

[13] Marion, G.H., et al. 2016, ApJ, 820, 92

[14] Milne, P.A., Foley, R.J., Brown, P.J., \& Narayan, G. 2015, ApJ, 803, 20

[15] Patat, N., et al. 2011, A\&A, 530, 63

[16] Patat, N., et al. 2012, A\&A, 545, 7

[17] Salpeter E. E., van Horn H. M., 1969, ApJ, 155, 183

[18] Scalzo, R.A., Ruiter, A.J., \& Sim, S.A. 2014, MNRAS, 445, 2535

[19] Shafter, A.W., et al. 2015, ApJS, 216, 34

[20] Shen, K.J., \& Bildsten, L. 2009, ApJ, 669, 1365

[21] Shen, K.J., \& Bildsten, L. 2014, 785, 61

[22] Tsebrenko D., Soker N., 2015, MNRAS, 447, 2568

[23] Zhu, C., Pakmor, R., van Kerkwijk, M.H., \& Chang, P. 2015, ApJ, 806, L1 